\title{
Long-term outcomes of mitral valve annuloplasty versus subvalvular sparing replacement for severe ischemic mitral regurgitation
}

\author{
Baotong Li, Hengchao Wu, Hansong Sun, Jianping Xu, \\ Yunhu Song, Wei Wang, Shuiyun Wang \\ State Key Laboratory of Cardiovascular Disease, Department of Adult Cardiac Surgery, Fuwai Hospital, \\ National Center for Cardiovascular Disease, Chinese Academy of Medical Science, \\ Peking Union Medical College, Beijing, China
}

\begin{abstract}
Background: Although practice guidelines recommend surgery for patients with severe chronic ischemic mitral regurgitation (CIMR), they do not specify whether to repair or replace the mitral valve. The purpose of this study was to evaluate the long-term outcomes in patients with severe CIMR undergoing mitral valve annuloplasty (MVA) versus subvalvular sparing mitral valve replacement (MVR).

Methods: 392 consecutive patients who underwent MVA or subvalvular sparing MVR for treatment of severe CIMR were retrospectively reviewed.

Results: After adjustment for baseline differences with multivariable regression analysis at 53 months follow-up (interquartile range, 34-81 months), there was no significant difference between the two groups for risk of major adverse cardiac or cerebrovascular events (MACCE), cardiac death, or all-cause death. Propensity score matching extracted 77 pairs. During the follow-up, compared with the MVR group, both the left atrium and left ventricle end-diastolic diameter were markedly larger $(p=0.013$ and $p=0.033$, respectively), and the incidence of mitral regurgitation recurrence was significantly higher in the MVA group $(p<0.001)$. No significant difference was observed between the two propensity score-matched groups in composite in-hospital outcomes, overall survival, freedom from cardiac death or MACCE, except subvalvular sparing MVR was associated with a lower incidence of hospitalization for heart failure than MVA $(p=0.015)$.

Conclusions: Subvalvular sparing MVR is a suitable management of patients with severe CIMR, it is more favorable to ventricular remodeling and is associated with a lower incidence of hospitalization for heart failure than MVA. (Cardiol J 2019; 26, 3: 265-274)

Key words: chronic ischemic mitral regurgitation, mitral valve annuloplast, subvalvular sparing mitral valve replacement, coronary artery bypass grafting
\end{abstract}

\section{Introduction}

Chronic ischemic mitral regurgitation (CIMR) is common and is associated with worse long-term survival and functional status [1]. It is generally agreed that severe mitral regurgitation (MR) requires mitral valve intervention, but the optimal management of patients with severe CIMR, specifically the choice between mitral valve annuloplasty (MVA) and mitral valve replacement (MVR), has long been debated [2-5]. To date, there are no prospective randomized trials evaluating

Address for correspondence: Hansong Sun, MD, PhD, State Key Laboratory of Cardiovascular Disease, Department of Adult Cardiac Surgery, Fuwai Hospital, National Center for Cardiovascular Disease, Chinese Academy of Medical Science, Peking Union Medical College, Beijing, China, tel: +86 010 88322351, fax: +86 01088322351 , e-mail: drsunhs@sina.com 
the long-term outcomes of MVA versus MVR for severe CIMR, while published series have provided a wide range of results for long-term outcomes. Considering the different conclusions which might have been derived from heterogeneity of patient cohorts and methods of treatment, the present study is a long-term design and propensity score (PS) matched analysis to evaluate the effectiveness of MVA versus sub-valvular sparing MVR for severe CIMR.

\section{Methods}

\section{Patients and study design}

This study was approved by the Human Research Ethics Committee of the Fuwai Hospital and was performed in accordance with the Declaration of Helsinki and approved guidelines. CIMR was defined by coronary angiographic and echocardiographic findings according to accepted criteria, i.e., 1) MR occurring more than 16 days after myocardial infarction; 2) type I/IIIb leaflet dysfunction following Carpentier's classification; and 3) 70\% or greater stenosis of at least one coronary artery, with wall motion abnormalities of the corresponding left ventricular (LV) segment [3].

Between January 2003 and December 2014, a total of 1040 patients with CIMR were hospitalized to undergo coronary artery bypass grafting (CABG) combined with MVA or MVR. From the initial cohort, 642 patients were excluded for various reasons [3], i.e., 1) Preoperative $M R \leq 2+$, congenital valvular heart disease, rheumatic or degenerative valvular disease, infective endocarditis, presence of aortic valve regurgitation or stenosis, emergency surgery, repeat operation; or 2) Performance of other procedures, such as LV reconstruction/reshaping, partial band/pericardial annuloplasty, or procedures other than mitral ring annuloplasty for treatment. Moreover, the patients who underwent MVR without preserving the subvalvular apparatus were excluded. In addition, 6 were lost to follow-up. Thus, the final study cohort comprised 392 patients: $306(78.1 \%)$ patients underwent MVA whereas 86 (21.9\%) underwent subvalvular sparing MVR.

Baseline patient characteristics, echocardiography data, operative data, and surgical techniques were collected from the division of cardiovascular surgery database and individual medical records. Patients were followed up through the internet or by telephone interview and outpatient department records.

\section{Surgical technique}

All surgical procedures were performed with standard bypass techniques through median sternotomy by senior surgeons with a special interest in mitral valve surgery. The decision to perform MVA or subvalvular sparing MVR was at the surgeon's discretion. Downsizing ring annuloplasty ( 2 sizes) was used in all patients subjected to MVA. The ring size was determined by measurements of the intertrigonal distance and anterior leaflet height. Intraoperative transesophageal echocardiography was routinely used. A successful MVA was defined as a leaflet coaptation of $\geq 0.8 \mathrm{~cm}$ and $M R \leq 1$ at transesophageal echocardiography performed at the end of cardiopulmonary bypass [3, 6]. Subvalvular apparatus were preserved when performing MVR, including posterior leaflet preservation, posterior and partial anterior leaflet preservation and both leaflet preservation. The decision to perform which kind of procedure was at the surgeon's discretion according to situational conditions. The posterior mitral valve leaflet was left intact in all patients undergoing MVR. In 8 of patients undergoing MVR, the middle portion of the anterior leaflet was resected and the remaining leaflet tissue was plicated with individual valve sutures. In 23 patients undergoing MVR, the anterior leaflet of the valve was partly or completely detached from the mitral annulus and divided in the middle at the 12 o'clock position, and the leftward portion of the anterior leaftlet was plicated to the anterolateral commissure with a pledgetted 4-0 polypropylene suture. The rightward a portion of the anterior mitral leaflet was similarly plicated to the posteromedial commissure. Complete revascularization was achieved in all patients with arterial conduits or saphenous vein grafts. All patients received the same perioperative care and medical therapy according to guidelines.

\section{Echocardiography}

Two-dimensional and Doppler transthoracic echocardiography examinations were performed before operations and at pre-discharge for all patients. MR was classified as mild (grade $1+$ ), moderate (grade $2+$ ), or severe (grades $3+$ and $4+$ ) [7]. LV inferior basal wall motion abnormality (BWMA) includes hypokinesia, dyskinesis and aneurysm. Echocardiographic criteria for aneurysm were evidence of thinning and localized LV dilation or distortion. Dyskinesis was the presence of outward displacement of the LV wall during systole $[8,9]$. 
Statistical analysis

All statistical analyses were performed by SPSS version 20 (IBM SPSS Inc., Chicago, IL), SAS software version 9.2 (SAS Institute) and Graph Pad Prism release 5 (Graph Pad Software Inc., La Jolla, Calif) statistical packages. All reported p values are two sided, and values of $\mathrm{p}<0.05$ were considered to indicate statistical significance. Continuous data are shown as mean \pm standard deviation. The Student $t$ test was used to measure the differences for variables with a normal distribution and equal variances. The Wilcoxon rank sum test was used for non-normally distributed variables. Categorical data are displayed as frequencies and percentages and comparisons were made with $\chi^{2}$ tests (Fisher exact tests if appropriate). A stepwise multivariable Cox proportional hazards model was developed to determine the independent risk factors. Variables with a $p$ value less than 0.10 in the univariate analyses were entered into multivariable models. Differences in risk-adjusted, long-term rates of study outcomes among patients who underwent different surgical procedures were assessed by the use of multivariable Cox proportional hazards regression with adjustment for all patient-level variables in Table 1. Cumulative event rates were calculated using a Kaplan-Meier method, and different event curves of outcomes were compared using a log-rank test.

To reduce the impact of treatment selection bias and potential confounding in the observational study, rigorous adjustment for baseline differences by use of propensity score matching was performed [10]. A PS representing the probability of having subvalvular sparing MVR as opposed to MVA was calculated for each patient by using a non-parsimonious multivariable logistic regression model. Variables used in the model are shown in Table 1. Pairs of patients with MVA and sub-valvular sparing MVR were matched using calipers of width 0.2 standard deviations of logit of the PS [11]. Model discrimination was assessed with $\mathrm{C}$ statistics, and model calibration was assessed with HosmerLemeshow statistics. Finally, 77 pairs of patients were matched to obtain risk-adjusted outcome comparisons between the two groups.

\section{Results}

\section{Patient characteristics}

The demographic, clinical and procedural data of patients who underwent MVA and subvalvular sparing MVR before and after PS matching are illustrated in Table 1. Before matching, patients who underwent subvalvular sparing MVR were older, with a worse mitral regurgitation grade and better left ventricular ejection fraction (EF).

Three kinds of complete symmetric rings were used in the present study, with the median size of $28 \mathrm{~mm}$ (interquartile range, $28-29 \mathrm{~mm}$ ): Duran Ancore (Medtronic, Santa Ana, CA), CarpentierEdwards Physio ring I (Edwards Lifesciences, Irvine, CA), Carpentier-Edwards Physio ring II (Edwards Lifesciences, Irvine, CA). There were seven types of prosthetic valves, with a median size of $27 \mathrm{~mm}$ (interquartile range, $27-29 \mathrm{~mm}$ ). The rate of bioprosthesis was $46.5 \%$ (40/86). Three types of bioprostheses were used $(n=40)$ : Mosaic (Medtronic, Santa Ana, CA), Carpentier-Edwards Perimount (Edwards Lifesciences, Irvine, CA) and Hancock II (Medtronic, Santa Ana, CA). Four types of mechanical valves were used $(n=46)$ : Medtronic Open Pivot (Medtronic, Minneapolis, $\mathrm{MN})$, On-X valve (On-X Life Technology, Austin, TX), CarboMedics Mechanical (Sorin-CarboMedics Inc, Italia, S.r.l) and St. Jude valve (St. Jude Medical, Minneapolis, MN). Subvalvular apparatus were preserved when performing MVR, with posterior leaflet preservation in $55(64.0 \%)$ patients, posterior and partial anterior leaflet preservation in $8(9.3 \%)$ patients, and both leaflets preservation in $23(26.7 \%)$ patients.

\section{Follow-up and outcomes}

The clinical follow-up was closed on January 1 , 2017. The median follow-up was 53 months (interquartile range, 34-81 months) with a completion rate of $98.5 \%$ (392/398) in the overall cohort. During follow-up, $62(15.8 \%)$ patients died, of whom $53(13.5 \%)$ died of a cardiac cause. The overall survival rates at 5 and 10 years were $86.6 \%$ and $52.9 \%$, respectively. Freedom from cardiac death at 5 and 10 years were $88.1 \%$ and $63.9 \%$, respectively.

After adjustment for baseline differences with Cox proportional hazard model analysis, there was no significant difference between MVA and subvalvular sparing MVR in risks of major adverse cardiac or cerebrovascular events (MACCE: cardiac death, repeat revascularization and myocardial infarction, stroke, subsequent mitral valve surgery, or hospitalization for heart failure), cardiac death, or overall death (for MACCE: $p=0.063$; for cardiac death: $\mathrm{p}=0.549$; and for overall death: $\mathrm{p}=0.759)($ Table 2).

\section{Risk factor analysis}

Multivariable analysis showed that age and preoperative $\mathrm{EF}$ were independent predictors of overall death (for age: hazard ratio [HR], 1.03; 95\% 


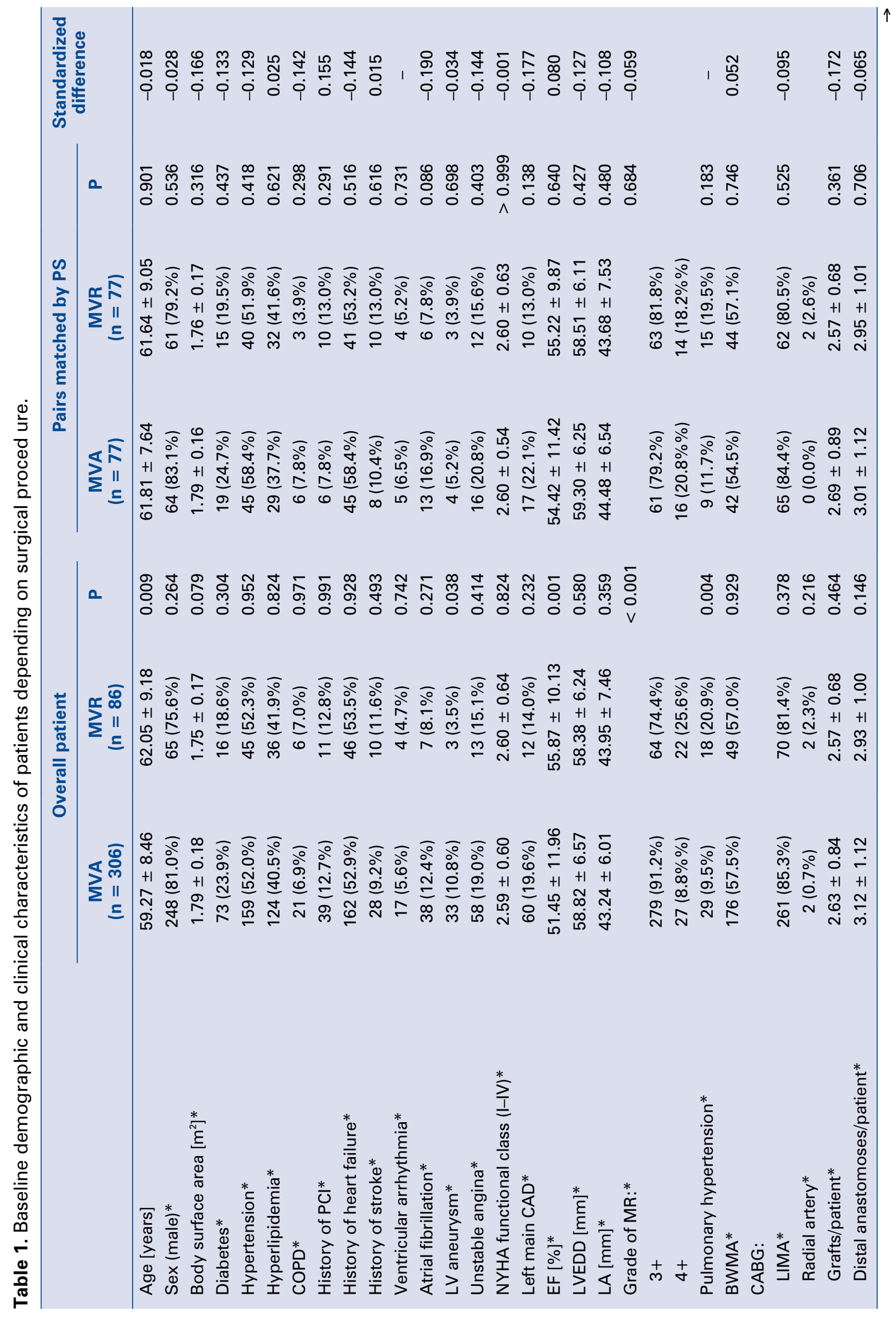




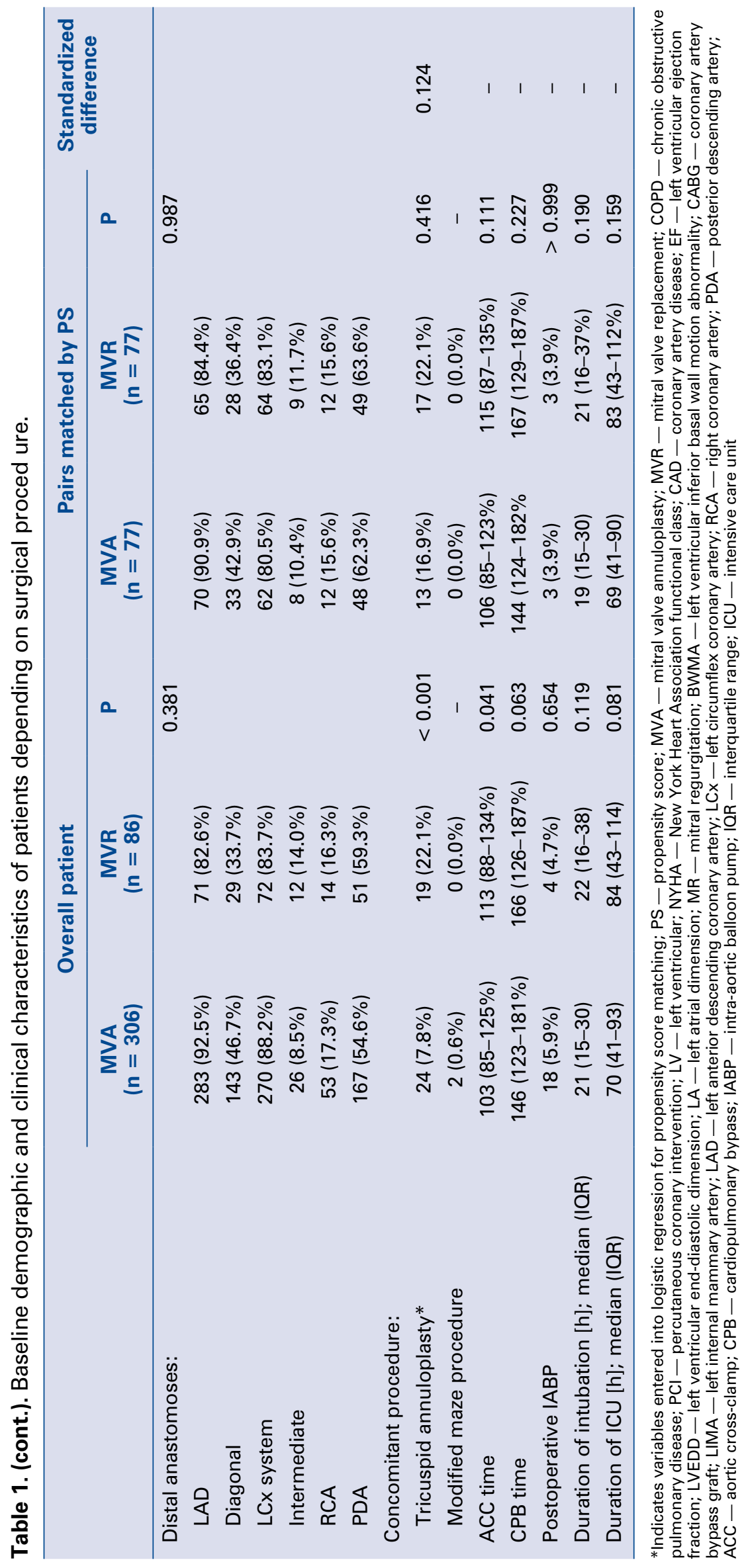


Table 2. Long-term outcomes according to different surgical procedures in the overall population.

\begin{tabular}{lcccc}
\hline & MVA & MVR & Adjusted HR H $^{\#} \%$ CI) & P \\
\hline All patients & 306 & 86 & & 0.549 \\
Cardiac death & $41(13.4 \%)$ & $12(14.0 \%)$ & $1.25(0.60-2.62)$ & 0.759 \\
Overall death & $50(16.3 \%)$ & $12(14.0 \%)$ & $0.90(0.44-1.82)$ & 0.063 \\
MACCE & $82(26.8 \%)$ & $14(16.3 \%)$ & $0.55(0.29-1.03)$ & \\
\hline
\end{tabular}

\#Multivariable Cox proportional hazard analysis was used with adjustment for all patient-level variables (Indicated by*) in Table 1. The HRs were reported for MVA with MVR as reference; HR - hazard ratio; $\mathrm{Cl}$ - confidence interval; MACCE - major adverse cardiac and cerebrovascular event; MVA - mitral valve annuloplasty; MVR - mitral valve replacement.

Table 3. Cox proportional hazard analysis for overall death and major adverse cardiac and cerebrovascular event (MACCE) at long-term follow-up.

\begin{tabular}{|c|c|c|c|c|}
\hline \multirow[t]{2}{*}{ Predictors } & \multicolumn{2}{|c|}{ Univariable } & \multicolumn{2}{|c|}{ Multivariable } \\
\hline & $\mathbf{P}$ & HR $(95 \% \mathrm{CI})$ & $\mathbf{P}$ & HR (95\% CI) \\
\hline \multicolumn{5}{|l|}{ Predictors of overall death: } \\
\hline Surgical procedures* & 0.895 & $0.96(0.51-1.80)$ & 0.997 & \\
\hline Age & 0.032 & $1.03(1.01-1.07)$ & 0.030 & $1.03(1.01-1.07)$ \\
\hline EF & $<0.001$ & $0.96(0.94-0.98)$ & $<0.001$ & $0.96(0.94-0.98)$ \\
\hline Grafts/patient & 0.045 & $1.41(1.01-1.96)$ & 0.243 & \\
\hline Anastomoses/patient & 0.083 & $1.24(0.97-1.57)$ & 0.351 & \\
\hline \multicolumn{5}{|l|}{ Predictors of MACCE: } \\
\hline Surgical procedures* & 0.119 & $0.64(0.36-1.12)$ & 0.260 & \\
\hline Age & 0.031 & $1.03(1.00-1.05)$ & 0.055 & \\
\hline History of heart failure & 0.010 & $1.72(1.14-2.60)$ & 0.337 & \\
\hline Ventricular arrhythmia & 0.028 & $2.17(1.09-4.31)$ & 0.064 & \\
\hline $\mathrm{EF}$ & $<0.001$ & $0.95(0.94-0.97)$ & $<0.001$ & $0.96(0.94-0.97)$ \\
\hline BWMA & 0.004 & $1.88(1.23-2.87)$ & 0.357 & \\
\hline Left ventricular aneurysm & 0.066 & $1.77(0.96-3.25)$ & 0.823 & \\
\hline Grafts/patient & $<0.001$ & $1.66(1.25-2.20)$ & 0.012 & $1.48(1.11-1.97)$ \\
\hline Anastomoses/patient & 0.004 & $1.33(1.10-1.61)$ & 0.875 & \\
\hline
\end{tabular}

*Indicates mitral valve annuloplasty or replacement; $\mathrm{HR}$ - hazard ratio; $\mathrm{Cl}$ — confdence interval; EF — left ventricular ejection fraction; BWMA - left ventricular inferior basal wall motion abnormality

confidence interval [CI] 1.01-1.07, $\mathrm{p}=0.030$; and for EF: HR 0.96; 95\% CI 0.94-0.98, p < 0.001), while the number of grafts and preoperative $\mathrm{EF}$ were independent predictors of MACCE (for the number of grafts: HR 1.48; 95\% CI 1.11-1.97, $\mathrm{p}=0.012$; and for EF: HR $0.96 ; 95 \%$ CI $0.94-0.97$, $\mathrm{p}<0.001)$. Of note, the choice of MVA or subvalvular sparing MVR was not a significant predictor of late overall death or MACCE ( $\mathrm{p}=0.997$ and $\mathrm{p}=0.260$, respectively) (Table 3 ).

Results of propensity score matching analysis

After PS matching, 77 pairs were extracted by 1:1 manner using nearest neighbor matching without replacement. Late deaths occurred in
29 patients, including 26 cardiac deaths. The 5 - and 10 -year overall survival rates were $80.9 \%$ and $55.8 \%$, respectively. The 5 - and 10 -year freedom from cardiac death rates were $82.5 \%$ and $62.1 \%$, respectively. There were no differences in preoperative and operative characteristics between the PS-matched patients (Table 1). The incidences of composite in-hospital outcomes (stroke, reoperation for bleeding, application of intra-aortic balloon pump and acute renal failure) were similar between the two PS-matched groups (Table 4). During follow-up, compared with the MVR group, both the left atrium and left ventricle end-diastolic diameter were markedly larger $(p=0.013$ and $\mathrm{p}=0.033$, respectively), and the incidence of MR 
Table 4. Early clinical outcomes of propensity score-matched patients.

\begin{tabular}{lccc}
\hline Variables & MVA $(\mathbf{n}=\mathbf{7 7})$ & MVR (n = 77) & P \\
\hline Composite in-hospital outcome & 9 & 15 & 0.183 \\
In-hospital mortality & $1(1.3 \%)$ & $2(2.6 \%)$ & 0.556 \\
Complications: & $8(10.4 \%)$ & $13(16.9 \%)$ & 0.240 \\
Stroke & $0(0 \%)$ & $0(0 \%)$ & - \\
Reoperation for bleeding & $1(1.3 \%)$ & $3(3.9 \%)$ & 0.300 \\
Postoperative IABP & $3(3.9 \%)$ & $3(3.9 \%)$ & $>0.999$ \\
Respiratory complication & $3(2.5 \%)$ & $5(7.4 \%)$ & 0.138 \\
Acute renal failure & $1(1.3 \%)$ & $2(2.6 \%)$ & 0.556 \\
\hline
\end{tabular}

MVA - mitral valve annuloplasty; MVR - mitral valve replacement; IABP — intra-aortic balloon pump

Table 5. Perioperative and follow-up echocardiographic results of propensity score-matched patients.

\begin{tabular}{|c|c|c|c|c|c|c|}
\hline \multirow[t]{2}{*}{ Variables } & \multicolumn{3}{|c|}{ MVA (n = 77) } & \multicolumn{3}{|c|}{ MVR (n = 77) } \\
\hline & Preoperative & Postoperative & Follow-up & Preoperative & Postoperative & Follow-up \\
\hline EF [\%] & $54.42 \pm 11.42$ & $52.81 \pm 8.68$ & $52.29 \pm 8.23$ & $55.22 \pm 9.87$ & $52.62 \pm 8.62$ & $51.95 \pm 9.58$ \\
\hline $\begin{array}{l}\text { LVEDD } \\
\text { mid-ventricle [mm] }\end{array}$ & $59.30 \pm 6.25$ & $51.02 \pm 6.61$ & $55.91 \pm 5.23$ & $58.51 \pm 6.11$ & $51.32 \pm 8.25$ & $53.75 \pm 6.99 *$ \\
\hline $\mathrm{LA}[\mathrm{mm}]$ & $44.48 \pm 6.54$ & $38.32 \pm 4.76$ & $45.34 \pm 5.82$ & $43.68 \pm 7.53$ & $39.34 \pm 7.66$ & $42.76 \pm 6.25^{*}$ \\
\hline Mitral regurgitation: & - & - & $41(53.25 \%)$ & - & & $2(2.60 \%)^{*}$ \\
\hline Moderate & - & - & $32(41.56 \%)$ & - & & $2(2.60 \%)$ \\
\hline Severe & - & - & $9(11.69 \%)$ & - & & $0(0 \%)$ \\
\hline Periprosthetic leak & - & - & - & - & & $1(1.30 \%)$ \\
\hline
\end{tabular}

${ }^{*} p<0.05$ vs. MVA; MVA - mitral valve annuloplasty; MVR - mitral valve replacement; EF - left ventricular ejection fraction; LVEDD - left ventricular end-diastolic dimension; LA — left atrial dimension

recurrence was significantly higher in the MVA group $(\mathrm{p}<0.001)$ (Table 5). There were no significant differences in overall survival, freedom from cardiac death or MACCE between the two groups (all $\mathrm{p}>0.05$ ), except for a higher incidence of hospitalization for heart failure in the PS-matched MVA group than in the subvalvular sparing MVR group $(\mathrm{p}=0.015)$ (Fig. 1).

\section{Discussion}

According to practice guidelines, both MVA and MVR are recommended treatments for correction of severe ischemic MR [12]. However, an optimal surgical approach to treatment of severe ischemic MR remains controversial. Clinical studies have suggested that repair is associated with lower perioperative morbidity and mortality but has a higher risk of recurrence, which confers with a predisposition to atrial fibrillation, heart failure, and readmission, whereas replacement provides higher periopera- tive mortality but better long-term correction with a lower risk of recurrence [13-15]. When MVR is required, chordal sparing is the preferred technique. Okita et al. [16] and David et al. [17] reported that the subvalvular apparatus preservation results in improved LV function and enhanced survival. Preservation of the mitral subvalvular apparatus led to better postoperative LV function and survival than those after apparatus removal.

In the present study, no difference was observed in the incidences of early mortality or postoperative complications between the two PS-matched groups. Published literature provides a wide range of results in terms of early outcomes. Several recent experiences found no significant difference between the two surgical managements, this is in accordance with the present observations [2, 18], whereas several studies showed that mitral valve repair is associated with lower operative mortality and postoperative complications [19, 20]. 


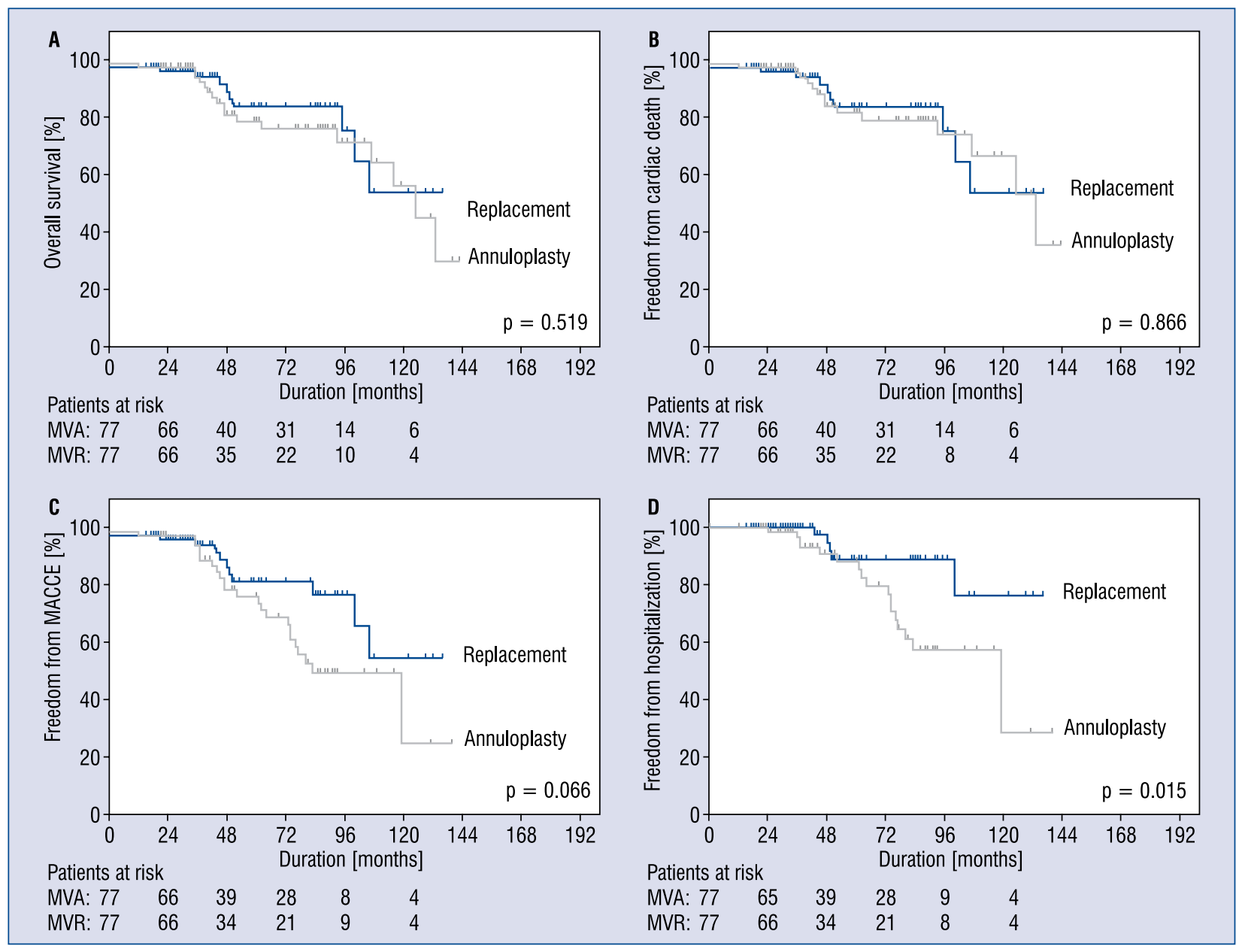

Figure 1. Kaplan-Meier curves for (A) overall survival (B) freedom from cardiac death (C) freedom from MACCE and (D) freedom from hospitalization for heart failure in 1:1 propensity score-matched mitral valve annuloplasty group (gray lines) and mitral valve replacement group (black lines); MACCE - major adverse cardiac and cerebrovascular event (cardiac death, repeat revascularization and myocardial infarction, stroke, subsequent mitral valve surgery, or hospitalization for heart failure); MVA - mitral valve annuloplasty; MVR - mitral valve replacement.

After adjustment for baseline differences with Cox proportional hazard model analysis, the present long-term observational study showed no substantial difference between the two managements of risk for MACCE, cardiac death, or overall death. Moreover, PS matching analysis also showed similar results. Follow-up echocardiographic results of PS-matched patients showed that, compared with the MVR group, both the left atrium and left ventricle end-diastolic diameter were markedly larger, and the incidence of MR recurrence was significantly higher in the MVA group. MVR provides a considerably more durable correction of MR than MVA [2, 19], which may have a beneficial effect on long-term outcomes. However, this effect must be weighed against any potential adverse consequences of a prosthetic valve, such as long-term thromboembolism, endocarditis, and structural valve deterioration [2]. The trial conducted by Goldstein et al. [2] showed that, at 2 years after either MVA or MVR for severe ischemic MR, there were no significant betweengroup differences with respect to LV reverse remodeling, however, the rates of MR recurrence were significantly higher in the MVA group than in the subvalvular sparing MVR group (58.8\% vs. $3.8 \%, \mathrm{p}<0.001$ ), related to heart failure and cardiovascular admissions [2]. Another important study carried out by Lorusso et al. [3] showed that 8 -year survival was $81.6 \% \pm 2.8 \%$ vs. $79.6 \% \pm$ $\pm 4.8 \%$ in MVA and MVR, respectively $(\mathrm{p}=0.42)$. Cohn et al. [21] reported a 5-year survival of 56\% and $91.5 \%$ in MVA and MVR, respectively, whereas a meta-analysis showed that the relative long-term 
risk of death was $35 \%$ higher in the MVR group than in the repair group [22].

Such differing conclusions might have been derived from the heterogeneity of patient cohorts. Therefore, in the present study, only patients undergoing MVA or MVR with complete myocardial revascularization were included, without congenital valvular heart disease, rheumatic valvular disease, infective endocarditis, presence of aortic valve regurgitation or stenosis, or having received other procedures. Moreover, the patients who underwent MVR without preserving the subvalvular apparatus were excluded. In addition, a propensity score model was constructed to minimize effects Limitation of confounding variables which ensured the reliability of study results.

\section{Limitations of the study}

First, this study reports retrospective data from a single center and is subject to all the limitations inherent to this design. The small study sample might have led to type II statistical errors. An appropriately powered, randomized, controlled trial evaluating the optimal management of CIMR would be useful inconfirming these results. Second, pre-, intra-, and postoperative information about the exact mechanisms and characteristics of MR were not available for all patients. For this reason, the objectives of this study were early and late outcomes. Third, selection bias should be introduced at the time of decision to perform surgical approaches because the decision to perform MVR or MVA may be related to the complexity of the patient and experience of the surgeon. To minimize the effects of selection bias, a propensity score model was constructed. Fourth, because of the 12 year inclusion time, there were three types of rings and seven types of prosthetic valves which could affect heterogeneity of the study. Another limitation is that, although this study assesses surgical approaches to the mitral valve, no detailed information was available regarding medical therapy at follow-up. However, with guideline-directed medical therapy by cardiologists, who had received systematic and standardized clinical training, the potential bias of therapy between groups is expected to be minimized.

\section{Conclusions}

The present study indicates that subvalvular sparing MVR was more favorable to ventricular remodeling and associated with a lower incidence of hospitalization for heart failure than MVA at follow-up. Therefore, subvalvular sparing MVR appears to be a suitable management for patients with CIMR undergoing mitral valve surgery and CABG. An appropriately powered, randomized, controlled trial evaluating the optimal management of CIMR would be useful in confirming the present results.

\section{Acknowledgements}

The authors thank Shanglin Chen (MD) for the assistance provided in the statistical analysis.

\section{Conflict of interest: None declared}

\section{References}

1. Bursi F, Enriquez-Sarano M, Nkomo VT, et al. Heart failure and death after myocardial infarction in the community: the emerging role of mitral regurgitation. Circulation. 2005; 111(3): 295-301, doi: 10.1161/01.CIR.0000151097.30779.04, indexed in Pubmed: 15655133.

2. Goldstein D, Moskowitz AJ, Gelijns AC, et al. CTSN. Two-Year Outcomes of Surgical Treatment of Severe Ischemic Mitral Regurgitation. N Engl J Med. 2016; 374(4): 344-353, doi: 10.1056/ NEJMoa1512913, indexed in Pubmed: 26550689.

3. Lorusso R, Gelsomino S, Vizzardi E, et al. Mitral valve repair or replacement for ischemic mitral regurgitation? The Italian Study on the Treatment of Ischemic Mitral Regurgitation (ISTIMIR). J Thorac Cardiovasc Surg. 2013; 145(1): 128-139, indexed in Pubmed: 23127376.

4. Di Salvo TG, Acker MA, Dec GW, et al. Mitral valve surgery in advanced heart failure. J Am Coll Cardiol. 2010; 55(4): 271-282, doi: 10.1016/j.jacc.2009.08.059, indexed in Pubmed: 20117430.

5. Perrault LP, Moskowitz AJ, Kron IL, et al. Optimal surgical management of severe ischemic mitral regurgitation: to repair or to replace? J Thorac Cardiovasc Surg. 2012; 143(6): 1396-1403, doi: 10.1016/j.jtcvs.2011.05.030, indexed in Pubmed: 22054660.

6. Vahanian A, Alfieri O, Andreotti F, et al. Joint Task Force on the Management of Valvular Heart Disease of the European Society of Cardiology (ESC) and the European Association for Cardio-Thoracic Surgery (EACTS), ESC Committee for Practice Guidelines (CPG), Joint Task Force on the Management of Valvular Heart Disease of the European Society of Cardiology (ESC), European Association for Cardio-Thoracic Surgery (EACTS). Guidelines on the management of valvular heart disease (version 2012): the Joint Task Force on the Management of Valvular Heart Disease of the European Society of Cardiology (ESC) and the European Association for Cardio-Thoracic Surgery (EACTS). Eur J Cardiothorac Surg. 2012; 42(4): S1-44, doi: 10.1093/ejcts/ ezs455, indexed in Pubmed: 22922698.

7. Zoghbi WA, Enriquez-Sarano M, Foster E, et al. American Society of Echocardiography. Recommendations for evaluation of the severity of native valvular regurgitation with two-dimensional and Doppler echocardiography. J Am Soc Echocardiogr. 2003; 16(7): 777-802, doi: 10.1016/S0894-7317(03)00335-3, indexed in Pubmed: 12835667.

8. Weyman AE, Peskoe SM, Williams ES, et al. Detection of left ventricular aneurysms by cross-sectional echocardiography. Circulation. 1976; 54(6): 936-944, indexed in Pubmed: 991409.

9. Lebeau R, Di Lorenzo M, Amyot R, et al. A new tool for estimating left ventricular ejection fraction derived from wall motion 
score index. Can J Cardiol. 2003; 19(4): 397-404, indexed in Pubmed: 12704486.

10. Li F, Zaslavsky AM, Landrum MB. Propensity score weighting with multilevel data. Stat Med. 2013; 32(19): 3373-3387, doi: 10.1002/sim.5786, indexed in Pubmed: 23526267.

11. Austin PC. Optimal caliper widths for propensity-score matching when estimating differences in means and differences in proportions in observational studies. Pharm Stat. 2011; 10(2): 150-161, doi: 10.1002/pst.433, indexed in Pubmed: 20925139.

12. Nishimura RA, Otto CM, Bonow RO, et al. 2017 AHA/ACC Focused Update of the 2014 AHA/ACC Guideline for the Management of Patients With Valvular Heart Disease: A Report of the American College of Cardiology/American Heart Association Task Force on Clinical Practice Guidelines. Circulation. 2017; 135(25): e1159-e1195, indexed in Pubmed: 28298458.

13. Al-Radi OO, Austin PC, Tu JV, et al. Mitral repair versus replacement for ischemic mitral regurgitation. Ann Thorac Surg. 2005; 79(4): 1260-1267. discussion -7., doi: 10.1016/j.athoracsur.2004.09.044, indexed in Pubmed: 15797060

14. Grossi EA, Goldberg JD, LaPietra A, et al. Ischemic mitral valve reconstruction and replacement: comparison of long-term survival and complications. J Thorac Cardiovasc Surg. 2001; 122(6): 1107-1124, doi: 10.1067/mtc.2001.116945, indexed in Pubmed: 11726886.

15. Gillinov AM, Wierup PN, Blackstone $\mathrm{EH}$, et al. Is repair preferable to replacement for ischemic mitral regurgitation? J Thorac Cardiovasc Surg. 2001; 122(6): 1125-1141, doi: 10.1067/ mtc.2001.116557, indexed in Pubmed: 11726887.

16. Okita Y, Miki S, Ueda Y, et al. Mitral valve replacement with maintenance of mitral annulopapillary muscle continuity in pa- tients with mitral stenosis. J Thorac Cardiovasc Surg. 1994; 108(1): 42-51, indexed in Pubmed: 8028378.

17. David TE, Ho WC. The effect of preservation of chordae tendineae on mitral valve replacement for postinfarction mitral regurgitation. Circulation. 1986; 74(3 Pt 2): I116-I120, indexed in Pubmed: 3742768.

18. Maltais S, Schaff HV, Daly RC, et al. Mitral regurgitation surgery in patients with ischemic cardiomyopathy and ischemic mitral regurgitation: factors that influence survival. J Thorac Cardiovasc Surg. 2011; 142(5): 995-1001, doi: 10.1016/j.jtcvs.2011.07.044, indexed in Pubmed: 21855899.

19. Dayan V, Soca G, Cura L, et al. Similar survival after mitral valve replacement or repair for ischemic mitral regurgitation: a metaanalysis. Ann Thorac Surg. 2014; 97(3): 758-765, doi: 10.1016/j. athoracsur.2013.10.044, indexed in Pubmed: 24370200.

20. Wang J, Gu C, Gao M, et al. Mitral valve replacement therapy causes higher 30-day postoperative mortality than mitral valvuloplasty in patients with severe ischemic mitral regurgitation: A meta-analysis of 12 studies. Int J Cardiol. 2015; 185: 304-307, doi: 10.1016/j.ijcard.2015.03.170, indexed in Pubmed: 25828670.

21. Cohn LH, Rizzo RJ, Adams DH, et al. The effect of pathophysiology on the surgical treatment of ischemic mitral regurgitation: operative and late risks of repair versus replacement. Eur J Cardiothorac Surg. 1995; 9(10): 568-574, indexed in Pubmed: 8562102.

22. Vassileva CM, Boley T, Markwell S, et al. Meta-analysis of shortterm and long-term survival following repair versus replacement for ischemic mitral regurgitation. Eur J Cardiothorac Surg. 2011; 39(3): 295-303, doi: 10.1016/j.ejcts.2010.06.034, indexed in Pubmed: 20727782 . 